\title{
Divinum opus est sedare dolorem
}

Ivo Casagranda

Direttore Responsabile

Ogni giorno, o quasi, per recarmi in Pronto Soccorso o in Medicina d'Urgenza, attraverso una scritta dorata sul pavimento di un corridoio risalente agli anni sessanta, che recita un detto attribuito a Ippocrate: «Divinum est sedare dolorem». Probabilmente quella frase è stata voluta per ricordare ai medici e agli infermieri che si recano al lavoro uno degli obblighi professionali più cogenti, che è quello dell'attenzione alla sofferenza delle persone, e rimanda, inoltre, a come il suo trattamento abbia qualcosa di trascendentale, tale da avvicinare chi la attenua o la elimina agli dèi.

Se così è, e se il lenimento della sofferenza, accanto all'allontanamento della morte attraverso la guarigione delle malattie, è stato una motivazione originaria della medicina, perché c'è ancora una resistenza da parte dei professionisti sanitari al suo trattamento e talvolta un rifiuto a ricevere le cure da parte dei pazienti? Qual è il peso delle influenze antropologiche, filosofiche e religiose sulla percezione del dolore e sul suo trattamento? Quanto l'incapacità, nei secoli, della medicina di lenire o togliere questo sintomo ha influenzato il comportamento e la cultura dell'uomo che ha cercato di trovare nella filosofia e nella religione le risposte?

Nel pensiero greco (Platone) il dolore è la conseguenza della disarmonia degli elementi ed è (Aristotele) un evento emotivamente negativo che contrasta la felicità. Nel pensiero romano lo stoicismo (Seneca) condanna tutte le emozioni ed esalta l'apatia come sopportazione del dolore.

$\mathrm{Nel}$ pensiero cristiano, la sofferenza è la conseguenza ineliminabile del peccato («partorirai nel dolore»), ma che assume il significato trascendentale della purificazione se vissuto cristianamente e in questa visione purificatoria è anche stato cercato e voluto (si pensi ai religiosi portatori di cilicio) fin quasi ai nostri giorni. Nel Medioevo la sofferenza viene vista come un elemento che unisce l'uomo alla passione di Cristo. Secondo Tommaso d'Aquino, l'unica e decisiva reazione al dolore è la contemplazione del Divino, mentre per Agostino, uno dei padri della Chiesa, esso è un mezzo di espiazione e di elevazione anche se scrive: «si possono accettare molti dolori, ma nessuno può essere amato».

Il pensiero cristiano è riuscito così a togliere l'uomo dalla disperazione dando un senso al dolore e, in una visione escatologica, rendendolo addirittura prezioso al fine del raggiungimento della santità. Pascal stesso lo considera una condizione naturale del cristiano, perché, proprio nella sofferenza, noi diventiamo uomini nella forma più autentica, cioè non più dipendenti dai beni materiali e dai piaceri terreni, quindi pronti alla relazione più stretta con Dio e alla sua conoscenza.

Il pensiero cristiano ha sicuramente influenzato, fino ai nostri giorni, l'atteggiamento nei riguardi di questa sgradevole esperienza sensoriale che per secoli è stata vista come naturale e necessario accompagnamento della malattia. Sicuramente, questa concezione ha influenzato anche l'atteggiamento dei medici e degli infermieri, basti pensare, ad esempio, ai ginecologi e alle loro difficoltà nell'eseguire il parto indolore, dovendosi confrontare con le parole bibliche sopra citate, secondo cui le donne avrebbero dovuto partorire nel dolore.

Il pensiero moderno desacralizza il dolore, che viene visto non come elemento e mezzo di redenzione, ma piuttosto come elemento da redimere e da eliminare, anche se momenti di idealizzazione permangono. Così, mentre per Hegel è un privilegio degli esseri più elevati e per Kant è ineliminabile dalla vita anche se riducibile con l'uso della ragione, per altri viene visto come un puro elemento biologico (Spinosa, Leibnitz). Con Schopenhauer si entra in una dimensione del pensiero che apre a quello contemporaneo: il dolore non è una realtà sensata che redime o che è da redimere, ma è un che di insensato che non ha giustificazione o spiegazione, anche se Giacomo Leopardi, suo contemporaneo, gli attribuisce ancora un significato molto alto nella vita dell'uomo, significato che può essere consolatorio per chi ne è affetto. Anche se più recentemente filosofi come Karl Jaspers hanno messo in guardia dall'idealizzare la sofferenza e se, nel nostro tempo alla metafisica del dolore si tende a sostituire la dimensione empirica, sulla base di ciò che la scienza ci dice, permane ancora all'interno della moderna medicina e della cultura contemporanea un pensiero duale caratterizzato, da una parte, dal voler togliere completamente il dolore dalla vita dell'uomo e, dall'altra, non riuscendoci completamente, dalla necessità di dare a questo un senso. Scrive Dietrich von Engelhardt che la medicina viene ripetutamente sfidata a riflettere sulla sua posizione fondamentale a cavallo tra le «scienze della natura» e le «scienze dello spirito», a recuperare la sua dimensione antropologica, quella cosmologica e quella metafisica. Proprio nell'incontro con il paziente il medico deve ca- 
larsi nella soggettività di questo, nella sua percezione del dolore e nella sua valutazione. Continua ancora von Engelhardt: «per la medicina e la sua terapia ha una grande importanza qual è il concetto di dolore che le sta alla base. Una tecnica della guarigione può definire il dolore come un guasto di una macchina mentre una cultura della guarigione partirà sempre dalla dimensione antropologica, cosmologica e anche metafisica del dolore».

Se adesso noi abbandoniamo queste riflessioni a contenuto filosofico, religioso e antropologico, che ci possono permettere di capire anche l'attuale atteggiamento di medici e infermieri nei riguardi della sofferenza fisica, e ci spostiamo su un piano di maggior concretezza per verificare cosa viene fatto nel mondo occidentale, e in particolare nel nostro Paese per affrontarla, sicuramente avremo molto materiale su cui meditare.

L'Organizzazione Mondiale della Sanità (OMS) ha molto insistito e molto insiste sulla necessità di trattare questo sintomo, prendendo addirittura come parametro dell'avanzamento sociale di una nazione il consumo di morfina in terapia. La Joint Commission on Accreditation of Healthcare Organisations (JCAHO) richiede, nei suoi standard di qualità, che tutti i pazienti vengano valutati per il dolore e che di conseguenza ne abbiano una trattamento adeguato. Anche l'Italia purtroppo ha un ritardo culturale, operativo e legislativo importante, anche se nel 2001 l'allora ministro Veronesi ha modificato e introdotto delle norme per un utilizzo burocraticamente meno complicato degli oppiacei.

Se noi ci spostiamo nel campo che ci è proprio, quello della medicina d'urgenza, per una valutazione su come questo sintomo venga gestito dai medici e dagli infermieri, ci rendiamo conto di quanta sia la strada da fare. Nonostante le raccomandazioni della JCAHO e delle società scientifiche, l'inadeguatezza del trattamento del dolore in Pronto Soccorso è ampiamente documentata e la percentuale di pazienti che non viene trattata è molto elevata. Dall'esame della letteratura si evince che da parte di questi ultimi vi è un'elevata aspettativa del controllo del proprio dolore in tale ambito; questa aspettativa riguarda il $72 \%$ dei pazienti con un $18 \%$ che richiede l'eliminazione totale dello stesso, anche se la richiesta di terapia non è sempre in rapporto all'intensità del dolore. Nonostante questa chiara aspettativa $e$ nonostante la richiesta esplicita da parte dei pazienti di essere trattati, l'atteggiamento dei medici d'urgenza e degli infermieri non è ancora adeguato.

Ad esempio, secondo alcuni lavori, meno del $40 \%$ dei pazienti con trauma viene trattato negli Emergency Departments americani e anche a molti di quelli con dolori d'altro genere non viene somministrata terapia o la stessa è inadeguata per tipo e dosaggio. In molti Pronto Soccorso non viene utilizzata nessuna scala di valutazione del dolore da parte della persona che ne è affetta e né questo né la sua valutazione vengono riportati in cartella. Ma per quali motivi succede ciò? Sicuramente tutti siamo figli ancora di una cultura che, come abbiamo visto precedentemente, trae origine da radici antiche e forti e su cui dovremmo ampiamente riflettere. Ma oltre a questo vi sono altri motivi, quali la presunzione di volere oggettivare un sintomo che non può essere oggettivato per cui, come dimostrato da alcuni studi, sia i medici sia gli infermieri stimano il dolore generalmente di minore intensità rispetto a come questo viene percepito dal singolo paziente. Oltre a questo, esistono altri miti, come quello che la scomparsa del dolore toglierebbe un elemento diagnostico o di valutazione successiva dell'andamento clinico (ad esempio nei casi di addome acuto) o di come l'utilizzo di oppiacei possa determinare dipendenze future.

Ma, al di là di questo, vi è un fatto oggettivo che è l'inadeguata preparazione tecnico-culturale dei medici e degli infermieri nella gestione del dolore. Tanabe e Buschmann, in un loro lavoro di sorveglianza, condotto nel 2000, avevano notato come per le infermiere la barriera principale al trattamento di questo sintomo fossero le scarse conoscenze e l'inadeguata preparazione e come, una volta sottoposte ad adeguata formazione, l'attenzione nella valutazione e nella gestione del dolore cambiasse sostanzialmente.

A tutt'oggi, sono pochi i percorsi accademici dello studio della medicina che al loro interno hanno dei momenti formativi volti alla gestione del dolore, e sono poche le società scientifiche che propongono a tale scopo linee guida o clinical pathway. Per migliorare la situazione, per operare secondo quanto la civiltà occidentale e la cultura del nostro tempo ci suggeriscono e per certi versi ci impongono, credo vada fatto ogni sforzo di tipo formativo, organizzativo e normativo (ad esempio rendendo più autonomi gli infermieri nell'utilizzo dei farmaci secondo protocolli concordati e validati) per gestire con più efficacia questo problema. Ritengo che ogni responsabile di Pronto Soccorso debba creare all'interno del proprio servizio una cultura condivisa sul trattamento del dolore attraverso l'utilizzo di strumenti come i protocolli. Per questo mi ha fatto piacere che a poca distanza dalla diffusione del primo numero della nostra rivista Emergency Care Journal, abbiamo ricevuto ben tre lavori che hanno come oggetto la gestione del dolore in Pronto Soccorso (due lavori sono pubblicati in questo numero mentre il terzo, infermieristico, verrà pubblicato prossimamente): ciò denota come questo problema cominci a esser sentito da medici e infermieri che lavorano nell'urgenza.

Desidero terminare questo mio editoriale con quanto scrive Paolo Tofanini, nel suo libro Eternit, Eterno, Eternità - Una storia di polvere, morte e speranze: le nostre a proposito del dolore da mesotelioma: «dicevo che la terapia del dolore è, per ora, l'unica prestazione sanitaria che deve essere incentivata, implementata e ampliata perché non c'è nessuna giustificazione nel far patire inutilmente le persone».

E questo pensiero credo vada assunto tale e quale anche nel nostro lavoro quotidiano di medici e infermieri che operano nel settore dell'urgenza, dove il dolore e la sofferenza sono davvero frequenti. 